\title{
Václav Jan Tomášeks Liederästhetik unter Berücksichtigung der Frage des Stils
}

\section{Václav Jan Tomášek's song aesthetics with the regard to the style}

Kateřina Alexandra Št'astná / katerina.alexandra@seznam.cz

Department of musicology, Faculty of Arts, Masaryk University, Brno, CZ

\begin{abstract}
This article aims to reflect the numerous and versatile song production by Václav Jan Tomášek (1774-1850) through the prism of its aesthetic and style aspects. Although Tomášek was interested in philosophy and aesthetic of fine arts, especially music, his views and ideas do not constitute a comprehensive system. Tomášek's songs mirrored influences of style of the Second Berlin as well as the Swabian and Wiennese Lieder School. There are commonalities between Tomášek's and Goethe's aesthetic and artistic concept of Lied. The significant and specific role in Tomášek's compositional language in this genre combines Classical structural features and more progressive elements (aperiodical melodic idioms, romantic colouring in harmony). Tomášek payd considerable attention to adequate musical setting of his chosen poems.
\end{abstract}

\section{Key words}

Václav Jan Tomášek, the nineteenth-century Lied, the aesthetic of Lied, poetry, Second Berlin School, Swabian Lieder School, Viennese Lieder School 


\section{Einführung}

An der Wende vom 18. zum 19. Jahrhundert und in der ersten Hälfte des 19. Jahrhunderts wirkte in der Hauptstadt des Königreiches Böhmen eine sehr bemerkenswerte und vielseitige Persönlichkeit der tschechischen klassischen und frühromantischen Musik, Václav Jan Tomášek (1774-1850). Er spielte nicht nur eine wichtige Rolle für die stilistische und künstlerische Entwicklung der tschechischen Musik, besonders wegen des Charakters seiner poetischen Klavierkompositionen und Lieder, sondern er beeinflusste auch besonders das Prager kulturelle und intellektuelle Leben. Hier sind vor allem seine pädagogische und publizistische Tätigkeit sowie seine in der nationalen, den Rahmen der Habsburgermonarchie aber respektierenden Aktivitäten hervorzuheben. Als Pädagoge erzog er zahlreiche Musiker, Pianisten und Komponisten. Zu seinen Schülern gehörten zum Beispiel der Pianist Alexander Dreyschock, die Komponisten Václav Jan Hugo Voř́išek, Josef Dessauer und František Max Kníže, oder der Musikkritiker und Ästhetiker Eduard Hanslick. Die von Tomášek im Jahre 1824 gegründete Lehranstalt für Klavierspiel und Komposition konkurrierte sehr erfolgreich mit ähnlichen Prager Institutionen - mit dem Prager Konservatorium und später auch der Orgelschule. Seine literarischen, publizistischen und kritischen Beiträge zur Kunst, vornehmlich Musik und Theater, waren im Prozess der Herauskristallisierung der musikalischen Kritik von Bedeutung. ${ }^{1}$ Noch bemerkenswerter als seine umfassenden Kenntnisse und intellektuellen Interessen ist die Tatsache, dass er seine musikalische Ausbildung sowohl auf dem Gebiet der pianistischen Fertigkeit und Virtuosität als auch in der Musiktheorie und Kompositionstechnik als Autodidakt zum höchsten Niveau brachte. Bei dieser Entwicklung seiner künstlerischen Persönlichkeit und der Selbstfortbildung stützte er sich auf die umfangreiche Basis von theoretischen Traktaten und didaktischen Abhandlungen.

Trotz seiner orchestralen Werke, deren unbestrittener geistiger Horizont und vollkommen beherrschte Kompositionstechnik ihn zum bedeutendsten tschechischen Symphoniker der Zeit vor Bedřich Smetana gemacht haben, ist Tomášek auf dem Gebiet des Liedes und des poetischen Klavierstückes höchst wahrscheinlich am progressivsten und für die neue Richtung und die weitere Entwicklung der Stiltendenzen der tschechischen Musik des 19. Jahrhunderts am wichtigsten. Das Komponieren von Liedern weist bei ihm eine Kontinuität auf, die von den frühen kompositorischen Versuchen über die ersten großen Erfolge mit der Vertonung von Gottfried August Bürgers Ballade Lenore die Aufmerksamkeit der musikliebenden Öffentlichkeit erregt und durch die er Anerkennung seiner schöpferischen Fähigkeiten gefunden hat, die durch die Lieder auf die neuentstandene tschechische Poesie, zu den Vertonungen von Gedichten Johann Wolfgang Goethes oder zu der einzigartigen musikalischen Fassung der altböhmischen Texte aus der Königinhofer Handschrift noch vergrößert wurde. In diesem Bereich seines künstlerischen Schaffens zeigen sich seine hervorragende Vertrautheit mit der

1 Siehe FUKAČ, Jiř́i. Václav Jan Křtitel Tomášeks früher Beitrag zur musikalischen Metakritik. In Studien zur Musikwissenschaft 49, 2002, S. 115-122. Zu diesem Tema auch PEC̆MAN, Rudolf. Tendenz zur Klassik: Betrachtungen über V. J. Tomášek. In Sbornik prací Filozofické fakulty brněnské univerzity, H 28, 1993, S. 55-67. 
deutschsprachigen und der neu entstehenden tschechischen Poesie sowie sein Sinn für die adäquate Vertonung der einzelnen Gedichte und die Abschattierung der stilistischen Nuancen sehr deutlich.

Der folgende Text bemüht sich, dass zahlreiche und vielfältige Liedschaffen von Václav Jan Tomášek im Kontext seiner ästhetischen und künstlerischen Standpunkte mit Berücksichtigung der Frage des Stils zu behandeln und auf die möglichen - direkten oder indirekten - musikalischen und ästhetischen Anknüpfungen an die sogenannte zweite Berliner, aber auch Wiener und Schwäbische Liederschule hinzuweisen. Aufmerksamkeit wird auch den Parallelen zwischen dem künstlerischen und ästhetischen Konzept des Liedes bei ihm und Goethe besonders bezüglich der Aussage der vertonten Poesie gewidmet.

\section{Tomášeks ästhetischen Grundlagen und Ansichten}

Obwohl sich Tomášek für die Fragen der Ästhetik der Kunst intensiv interessiert und bei seiner schöpferischen Arbeit stets auch auf die ästhetische Seite Rücksicht genommen hat, bilden seine Ideen und Ansichten keine komplexere Theorie oder ein Lehrgebäude. Dank seiner umfangreichen Ausbildung in der Philosophie und Literatur und der auBerordentlichen Breite seiner Interessen verfügte er über einen bemerkenswerten Überblick über diese Problematik. Bei der Gestaltung seiner ästhetischen Anschauungen ging er vor allem von den philosophischen und ästhetischen Richtungen aus, die sich gegen Ende des 18. und zum Anfang des 19. Jahrhunderts in Prag verbreiteten. Die Einflüsse der Nachahmungsästhetik haben sich dabei mit der Auffassung des Ausdruckprinzips des Klassizismus und mit den Elementen der Gefühlsästhetik der Romantik vereinigt. Während seines Studiums an der Prager Universität besuchte er $\mathrm{u}$. a. die Vorlesungen Karl Heinrich Seibts (Metaphysik), August Gottlieb Meissners (Ästhetik) oder Ignac Cornovas (Weltgeschichte). Inspirative Impulse gaben ihm auch die Prager öffentlichen Vorträge Joseph Georg Voglers. Zur Ausprägung seiner ästhetischen Ansichten war der Kontakt mit seinem Freund, dem Professor der Ästhetik an der Prager Universität Johann Heinrich Dambeck, von Bedeutung. Dambeck ging in seinem ästhetischen Konzept von der Philosophie Kants aus, war aber auch durch die Ideen der Romantiker Wilhelm Heinrich Wackenroder und Ludwig Tieck beeinflusst. ${ }^{2}$

Obwohl Tomášek in seiner Selbstbiographie seine ästhetischen Urteile über die Kunst keineswegs selten geäußert hat - besonders dann, wenn es sich um musikalische Werke und verschiedene Aufführungen handelte - kann man hier nur wenige nähere Bemerkungen finden, die sich auf das Liedschaffen anderer Komponisten oder auf sein eigenes ästhetisches und künstlerisches Konzept in diesem Genre beziehen. Dass er jedoch auf diesem Gebiet der vokalen Komposition und dessen aktueller Entwicklung

2 Näher zu dieser Problematik cf. VÍT, Petr. Estetické myšlení o hudbě. Praha, Academia 1987; víT, Petr. Zur Frage der Abwandlungen der musikästhetischen Begriffe in den böhmischen Ländern. Acta musicologica 60, fasc. 2, 1988, S. 195-204. 
die entsprechende Aufmerksamkeit gewidmet hat, beweisen seine beständigen und zahlreichen einschlägigen Kompositionen. Von der akribischen Präzision, die er für die Liedkomposition aufgewendet hat, zeugt die einige Jahre andauernde Vorbereitung, die der Vollendung seiner Vertonung von Bürgers Lenore vorangegangen war: Seine Arbeit an dieser Komposition hatte er im Sommer 1786 begonnen und ihre definitive Version erst im Jahre 1805 publiziert. Er selbst bemerkte dazu in seiner Selbstbiographie zum Jahre 1786: „In der Ferienzeit blieb ich in Prag und machte mich über die Komposition der Lenore von Bürger mit Begleitung des Pianoforte, von der ich einige Strophen zu Stande brachte; ich überzeugte mich aber bald, daß mir die erforderlichen Kenntnisse dazu noch fehlen, um ein solches Werk mit gleichem ungeschwächten Interesse durchführen zu können."

Der Erfolg, den ihm im Jahre 1805 die Veröffentlichung der Lenore brachte, belegt, dass er schon als junger Künstler auf der herrschenden Beliebtheit von Balladen flexibel und eigenständig zu reagieren wusste und eine beträchtliche Übersicht über die Liedproduktion seiner Zeit hatte. Eine nicht unbedeutende Rolle spielte dabei auch seine außerordentliche Vertrautheit mit einschlägigen theoretischen Abhandlungen. Er befasste sich mit dem intensiven Studium verschiedener, auf die Musik orientierter Schriften und Traktate, insbesondere im Zusammenhang mit der Arbeit an seinem eigenen geplanten System der Harmonie. ${ }^{4}$ Viele von diesen Abhandlungen behandeln theoretische Erörterungen zur Vokalmusik im Rahmen der ästhetischen Tradition des 18. Jahrhunderts. Auch in diesen Quellen konnte Tomášek also verschiedene Impulse für seinen künstlerischen Ausdruck finden. Der mehrteilige Charakter der Struktur in der Fassung von Bürgers Lenore von Tomášek sowie die darin angewandten musikalischen Mittel weisen einige gemeinsame Züge mit den Balladen von Carl Friedrich Zelter und Johann Rudolph Zumsteeg auf. Zelter war, wie bekannt, ein wichtiger Vertreter der zweiten Berliner Liederschule, während Zumsteeg zu den bedeutendsten Persönlichkeiten der sogenannten Schwäbischen Liederschule gehört. ${ }^{5}$ Tomášek hatte auch gute Kenntnisse des Kunstliedes Wiener Provenienz, was aus den Erwähnungen in seiner Selbstbiographie klar hervorgeht. Er hat seine Meinung zum künstlerischen Wert der Liedkompositionen anderer Autoren nur zweimal geäußert, und zwar im Falle von Joseph Haydn und Ludwig van Beethoven. Die Kunstlieder Josef Haydns fanden bei ihm volle Anerkennung. Er erwähnt besonders dessen nachahmenswerte musikalische Umsetzung des Textes und schrieb im Zusammenhang mit Haydns Lied „Die Treue“, ${ }^{6}$ dass es zu dem Ergreifendsten der Liederliteratur gehöre: „Auch Lieder kenne ich von ihm, die in der Auffassung des Textes als Muster für alle Tonsetzer der Erde gelten können. Ich erwähne hier nur des Einen zu dem Texte, Du Treue, kennst nicht meinen Schmerz', bist weit entfernt von mir" das zu dem Ergreifendsten im Liederfache gehört. Von eben so drastischer Kraft ist dessen Sturm für Chor mit Orchester."

3 TOMÁŠEK, Václav Jan. Selbstbiographie I. Libussa 1845, S. 372-373.

4 Ebenda.

5 Zumsteegs Lenore ist wahrscheinlich im Jahre 1797 entstanden.

6 D. h. „The Fidelity“/ „Die Treue“ aus: Sechs englische Kanzonetten, Hob. XXVla/6.

7 TOMÁŠEK, Václav Jan. Selbstbiographie II., Libussa, 1846, S. 332. 
$\mathrm{Zu}$ Beethovens Liedern schrieb Tomášek in einer definitiven Begründung seines Standpunkts zu den vokalen Kompositionen des Meisters: „Ich hörte der geistreichen Musik mit gespannter Aufmerksamkeit zu, denn mir lag viel daran, zu wissen, ob mein über Beethoven gefälltes Urtheil vielleicht etwa zu schroff sei, als ich an ihm schon früher keinen glücklichen Tonsetzer für den Gesang erkannte. Wie groß war dann meine Freude, daß ich ihm nicht Unrecht that, worüber mich bald eine nähere Bekanntschaft mit seinen Liedern, Messen und dem sollennen Oratorium ,Christus am Oelberge" ganz beruhigte. [...]."

Die Liedkompositionen Beethovens waren ihm also bekannt. Er hat sich aber leider nicht näher geäußert, welche Mängel er in dessen Liedern gesehen hat. Über seine Einwände könnte man nach seiner allgemeinen Meinung zu Beethovens Schaffen urteilen: „Ich halte ihn für einen der begabtesten Tondichter, jedoch nur für Instrumental-Musik, nicht aber für Vocal-Musik, worin er nicht sehr glücklich war. Die Harmonie, der Kontrapunkt, dann Eurythmie und vorzüglich die musikalische Aesthetik schienen ihm nicht allzusehr am Herzen zu liegen, daher selbst seine großangelegten Tonwerke durch manches Triviale entstellt sind." ${ }^{9}$

Obwohl Tomášek in seiner Selbstbiographie keine näheren Bemerkungen zu den Liedern von Wolfgang Amadeus Mozart gemacht hat, drücken alle seine Äußerungen über ihn die ausnahmslose Bewunderung für dessen Werke aus. Er sah in ihnen die vollkommene Erfüllung der höchsten künstlerischen Ideale in Übereinstimmung mit den Regeln der Ordnung, mit dem Respekt vor der Reinheit der Gattung und der Harmonie, mit der Wahl der Kompositionsmethoden und Ausdrucksmittel. Nach diesen künstlerischen und ästhetischen Idealen, die den Forderungen der Ästhetik des späten musikalischen Klassizismus entsprechen, hat Tomášek seiner schöpferischen Tätigkeit stets gestrebt.

\section{Die ästhetischen Aspekte von Tomášeks Liedschaffen}

Tomášek hat sich in den Angaben zu seinen Liedern meistens nur auf einige grundlegenden Informationen beschränkt, wie z. B. über den Namen des Verfassers des Textes, ob mehrere Sprachvarianten der unterlegten Worte zur Verfügung stehen, über den Verleger oder die Nummer in seinem Kompositionsverzeichnis. Nur einmal gibt er uns einen näheren Einblick in seine schöpferische Arbeit, und zwar im Falle einer Ballade von Karoline Pichler. ${ }^{10}$ Er verteidigte und erläuterte die hier von ihm angewandte Kompositionsmethode und die verwendeten musikalischen Mittel: „Die fromme Sage, die zu einem Bilde den Stoff gab, das heut noch in einem Gange des Stiftes hängt, wurde von Karoline Pichler zu einer Ballade benutzt, die ich, vom Herrn Prälaten aufgefordert, in Musik setzte. Von jeher der musikalischen Malerei nicht sehr hold mußte ich doch dießmal meine Zuflucht zu ihr nehmen, wenn ich der Dichtung nicht untreu sein wollte, und da die Ballade eine sehr umständliche Beschreibung eines grimmigen Gewitters enthält, worin der Donner und Blitz einander

8 Ebenda, S. 358.

9 TOMÁŠEK, Václav Jan. Selbstbiographie I., Libussa, 1845, S. 375.

10 Die Entstehung der Cisterzienser Abtey Hohenfurth in Böhmen, Ballade, op. 62 (1817). 
ablösen, was konnte mir, dem Tondichter, wohl anderes übrig bleiben, als der Musik einen solchen Charakter und Ausdruck zu geben, wodurch der Zuhörer in eine ähnliche Gemüthsstimmung mit Rosenberg ${ }^{11}$ versetzt, sich in dessen schreckenvolle Lage recht hinein fühlen könnte, was ich, ohne Natur nachahmen zu wollen, nie erreicht hätte. Es handelte sich daher, ehe ich an diese Composition ging, das Rhytmische in dem besonderen Rhythmus eines Gewitters zu erspähen, wozu sich nie eine so günstige Gelegenheit darbot, als in dem Jahr, wo ein Gewitter das andere gleichsam verfolgte. Auch würde ich kaum wo anders einen geeignetern Standpunkt gefunden haben, als hier, wo ich vom Herrengarten das weite Thal, Wienau genannt, mit seinem ungeheueren Horizont ganz übersehen und jedes Gewitter von seinem Beginn bis zu dessen Ende genau beobachten konnte. Daß ich es nie verabsäumte, mit Regenschirm unter dem Arm mich stets dahin zu begeben, und daß ich daran wohlgethan, zeigte sich bald, als ich die in Gratzen bereits angefangene und nach meiner Rückkehr in Prag erst vollendete, dem Stifte Hohenfurth gewidmete Ballade herausgab, welcher allgemeine Anerkennung zu Theil wurde."12

An Tomášeks Schilderung kann man erkennen, wie wichtig für ihn die entsprechende musikalische Fassung des Inhalts war. Er scheute dabei auch nicht davor zurück, ihm eher fremde stilistische Elemente zu benutzen, soweit sie zum angemessenen musikalischen Ausdruck des Textes etwa beitragen konnten. Die Tonmalerei verwendet er also nicht als bloße Nachahmung der Natur im Geiste der Nachahmungsästhetik, sondern als einen Weg, die von ihm beabsichtigte emotionelle Stimmung hervorzurufen. Darin wird der Einfluss des Ausdrucksprinzips der Spätklassik sowie, insbesondere in der Beziehung zur vertonten Poesie, eine gewisse Neigung zur romantischen Gefühlsästhetik ersichtlich.

Die Poesie diente Tomášek nicht nur als Quelle der Inspiration und der zur musikalischen Fassung geeigneten Texte. Nach seiner Meinung wirkte die Poesie in allen Gestalten des Lebens und allen Kunstformen. Er hielt sie für das grundsätzliche Element des künstlerischen Schaffens, wie sein Neffe Karl Viktor Hansgirg in seiner Reminiszenz an V. T. im Jahre 1855 schrieb:

„Er wurde von der Ansicht geleitet, daß die ,Poesie“ nicht blos in der großen Weltschöpfung und in allen Gestallten des Lebens, sondern daß sie auch in allen Kunstformen wirke, und er stellte darum für jeden Künstler als kategorischen Imperativ, er müsse ,Poet“ sein. Daher erfüllte ihn auch die Dichtkunst mit heiligem Schauer von ihrer tragischen und heroischen, und mit kindlicher Freude und zarter Wehmuth von ihrer lyrischen Seite. Insbesondere ließ er sich von dem echtlyrischen und in seiner äußersten Form sanggerechten Goethe anziehen, dessen Frische und Gesundheit seiner unmittelbaren Empfindung er so gut zu schätzen verstand, daß Goethe selbst den Compositionen seiner Lieder einen ungemein hohen Werth beigelegt hatte. [...] Sein GoetheEnthusiasmus übernahm sich indeß nicht zum Nachtheil des zweiten der beiden Dioskuren, ich meine Friedrich v. Schiller's. Das Pathos seiner glutvollen, großen Gefühle suchte er in einigen Liedercompositionen, dann aber auch in der Bearbeitung der ,Braut von Messina wiederzugeben. Es schien ihn jedoch bei der Tonsetzung manches Schiller'schen Gedichtes weniger die eigene Intuition, als vielmehr der Drang nach dem Experimente geleitet zu haben, ob man der Antithese

11 Die Zisterzienserabtei „Unser lieben Frau“ in Vyšší Brod/Hohenfurth wurde von Petr Wok von Rosenberg im Jahre 1259 gegründet. Einer Legende nach geschah dies aus Dankbarkeit nach dessen Rettung aus dem Fluss Moldau, als er sich mit seiner Bitte um Hilfe an die Madonna gewendet hat.

12 TOMÁŠEK, Václav Jan. Selbstbiographie III. Libussa, 1847, S. 441. 
Schiller'scher und Goethe'scher Lyrik nicht auch eine Parallele der Musik liefern und ob man übrigens auch die Schwierigkeiten bewältigen könne, welche der Tondichter theils in dem abstracten und philosophischen Geist, theils in dem breiten schwerfallenden Versbau der Schiller'schen Gedichte zu bewältigen findet."13

Tomášek hat die poetischen Texte für seine Kompositionen mit großer Sorgfalt und mit treffsicherem Geschmack ausgewählt. Dies und seine Wahl der Dichter sowie die Mannigfaltigkeit der vertonten Verse zeugen von seiner beachtlichen Vertrautheit mit der deutschsprachigen und neu entstandenen tschechischen Literatur.

Was die deutsche bzw. die deutschsprachige Poesie anbelangt, vertonte Tomášek neben Dichtern wie Ludwig Christoph Heinrich Hölty, Gottfried August Bürger, Christian Fürchtegott Gellert, Christoph August Tiedge, Friedrich Gottlieb Klopstock, Heinrich Heine, Friedrich Rückert, Ludwig Tieck, Karl Egon Ebert oder Karoline Pichler insbesondere die Gedichte von Johann Wolfgang Goethe. Eine spezifische Stellung nehmen bei ihm auch die musikalischen Fassungen einiger Werke von Friedrich Schiller ein.

In Hinsicht des Stils der von ihm ausgewählten poetischen Texte wählte er oft Gedichte, die unter dem Einfluss der Empfindsamkeit und der Sturm und Drang-Epoche standen. Auch die Komponisten der Zweiten Berliner Liederschule haben sehr oft Texte von Klopstock, Hölty, Schiller oder Goethe vertont.

In den Liedern zu den tschechischen Texten, die etwa 30 Prozent seines Schaffens in diesem Genre bilden, vertonte Tomášek am häufigsten Verse von Václav Hanka, Vojtěch Nejedlý, Antonín Marek oder Karl Egon Ebert. Einen erheblichen Teil von seinen vier tschechischsprachigen Liedersammlungen nimmt die im Volkston geschriebene Poesie Hankas ein. Hier schloss sich an die ästhetischen Fragen der Auswahl der Poesie auch der Aspekt des nationalen Bewusstseins an. Tomášek lebte und wirkte in der Zeit der Renaissance seines Volkes, der Geburt der modernen tschechischen Gesellschaft, Kunst und Kultur. Die wiedergeborene tschechische Sprache fand nach und nach ihren poetischen Ausdruck. Sie musste aber die Hindernisse überwinden, die für sie die gewaltsame Unterbrechung ihrer natürlichen Entwicklung im Zusammenhang mit der Germanisierung bedeutete. ${ }^{14}$ Tomášek glaubte aber an die Lebensfähigkeit und an das künstlerische Potenzial seiner Muttersprache. Im Vorwort zu Šestero pisniv hudbu uvedených (Sechs böhmische Lieder in Musik eingeführt) op. 48 aus dem Jahre 1813 schrieb er vom Wohlklang der tschechischen Sprache und von ihrer Eignung für die Verbindung mit der Musik. Er erkannte ihren eigenen ästhetischen Wert an: „[...] Ein Glück, daß unsere Schriftsteller fremde Sprachen beherrschend die Gründlichkeit und klingende Lieblichkeit ihrer Sprache erkannten und sich davon überzeugten, daß die Sprachen der Ausländer die Anmut nur von der Musik entlehnen; die böhmische Sprache verbindet aber, in Musik gesetzt, Süße mit Süße. "15

Die Vertonung der „originalaltböhmischen“ Texte der Königinhofer Handschrift hat ihm ohne die Belastung durch konventionelle Kompositionsvorgänge den Versuch eines

13 HANSGIRG, Karl Viktor. Reminiszenz an V. T. Libussa, 1855, S. 206-207.

14 Näher zu dieser Problematik siehe VYSLOUŽIL, Jiří. Václav Jan Křtitel Tomášeks Stellung in der tschechischen Musikkultur. In Sbornik praci Filozofické fakulty brněnské univerzity, 1975, S. 7-18.

15 TOMÁŠEK, Václav Jan. Šestero písni v hudbu uvedených. Sechs böhmische Lieder in Musik eingeführt, op. 48. K. V. Enders, 1813. 
neuen schöpferischen Ausdrucks auf dem Gebiet des Kunstliedes ermöglicht. Auch in diesem Falle ist es der Charakter des Textes, der bei ihm die Wahl der künstlerischen Ausdrucksmöglichkeiten und der progressiveren Melodik beeinflusst hat. Obwohl er in diesen seinen Liedern gegen einige Regeln der betonten Deklamation der tschechischen Sprache verstoßen hat - was Milena Vysloužilová in ihrer Studie Václav Jan Tomášeks Lieder zu tschechischen Texten für einen ästhetischen Mangel hält -, mindert das nicht die Bedeutung dieser Kompositionen für die stilistische und künstlerische Ausrichtung auf dem Gebiet des tschechischen Kunstliedes. ${ }^{16}$

\section{Die übereinstimmenden Aspekte der Liedästhetik bei Goethe und Tomášek}

Vergleicht man die intellektuellen und künstlerischen Richtungen der Persönlichkeit J. W. Goethes und V. J. Tomášeks, so kann man zahlreiche wesentliche gemeinsame Züge finden. Für beide war die ungemeine Breite und Allseitigkeit ihrer Ausbildung bezeichnend, beide gingen von der aufklärerischen Philosophie Kants aus, beide interessierten sich für die ästhetische Wahrnehmung des Kunstwerkes, für die Theorie der Musik und ihre Anwendung auf die Praxis. Sowohl Goethe als auch Tomášek legten großen Wert auf die adäquate musikalische Fassung der poetischen Texte, Goethe als Dichter und Autor der vertonten Poesie, Tomášek als schöpferischer Musiker. Für Goethe war die Musik die unerschöpfliche Quelle der Inspiration für seine literarischen Werke. Tomášek fand in der Poesie einen wesentlichen Ausgangspunkt für sein musikalisches Schaffen vornehmlich auf dem Gebiet des Kunstliedes und der poetischen Klavierkompositionen, Eklogen, Dithyramben und Rhapsodien. Beide haben die schöpferische Genialität Mozarts bewundert und seine Werke hochgeschätzt. Beide nahmen aber eine eher zurückhaltende oder kritische Stellung zu den stilistischen und formal progressiven Kompositionsmethoden bei Beethoven ein.

Obwohl Goethe selbst nicht komponiert und dem praktischen Musizieren sich nur sporadisch gewidmet hat, war sein Leben doch mit der Musik eng verbunden. Er pflegte zahlreiche und vielfältige Kontakte zu namhaften Komponisten und Tonkünstlern, verfasste Libretti für verschiedene musikalische Bühnenwerke, war als Leiter des Weimarer Theaters auch für die Opernaufführungen verantwortlich. Er sammelte Volkslieder (ähnlich wie Tomášek) und befasste sich mit Forschungen auf dem Gebiet der Akustik und der musikalischen Anthropologie. Er trug auch zur Entwicklung der Musikgeschichte bei. Dank der außerordentlichen Mannigfaltigkeit seines literarischen Schaffens, das von Adaptationen der Volkspoesie, über Liebeslieder, Oden, Balladen, Dithyramben und Hymnen zu philosophischen und hermeneutischen Werken reicht, wurde Goethe bald einer der meistvertonten deutschen Dichter, besonders auf dem Gebiet des Kunstliedes, für dessen ästhetische und künstlerische Auffassung er sich intensiv interessierte.

16 Siehe VYSLOUŽILOVÁ, Milena. Václav Jan Tomášeks Lieder zu tschechischen Texten. In Sborník prací Filozofické fakulty brněnské univerzity, H9, 1974, S. 47-64. 
Aufgrund seiner eigenen schöpferischen Absichten und der Diskussionen mit Komponisten, mit welchen er in engerem Kontakt stand, formulierte er seine eigene ästhetische und künstlerische Konzeption des Kunstliedes. Zu seinen einflussreichsten musikalischen Ratgebern gehörten zuerst Philipp Christoph Kayser, ab1789 Johann Friedrich Reichardt und ab dem Jahre 1796 Carl Friedrich Zelter, beide Vertreter der Zweiten Berliner Liederschule. Obwohl diese aus der Tradition der Ersten Berliner Liederschule hervorgegangen war, waren ihre ästhetischen und künstlerischen Ausgangspunkte einigermaßen andere. Der Schwerpunkt der Liederkomposition liegt bei ihnen immer in der angenehmen, volkstümlich konzipierten Vokalmelodie, das Klavier spielte die Rolle des Begleitungsinstruments.

Die ästhetische Konzeption des Kunstliedes bei Goethe hat viele übereinstimmende Aspekte mit den ästhetischen und künstlerischen Forderungen der Zweiten Berliner Liederschule. Goethe bevorzugte eindeutig das Strophenlied gegenüber einer von ihnen durchkomponierten Variante. Er forderte eine einfache, angenehme Melodie der Singstimme mit einer unkomplizierten Klavierbegleitung. Die Gestaltung der musikalischen Fassung sollte nicht die Aufmerksamkeit des Zuhörers von der vertonten Poesie ablenken. Die Schlüsselrolle spielte dabei die adäquate Interpretation des Vokalparts und der ausdruckvolle Vortrag des gesungenen Gedichtes, die den Inhalt mit den entsprechenden Abschattierungen und Nuancen spiegeln sollten. ${ }^{17}$

Der Respekt vor der Form und den Inhalt des Gedichtes ohne die übermäßige Ausnutzung der imaginativen musikalischen Mittel, die Goethe zum Beispiel auch in Schuberts Vertonung der Ballade Erlkönig für störend hielt, und gefällige, angenehme Melodik waren auch für Tomášeks eigenen musikalischen Ausdruck in seinen Liedern bezeichnend.

Wie Tomášek in seiner Selbstbiographie schrieb, sagte Goethe nach dem Anhören einiger von dessen Vertonungen seiner Gedichte: „Sie haben das Gedicht verstanden“, und weiter „ich kann nicht begreifen, wie Beethoven und Spohr das Lied gänzlich mißverstehen konnten, als sie es durchkomponierten; die in jeder Strophe auf derselben Stelle vorkommenden gleichen Unterscheidungszeichen wären, sollte ich glauben, für den Tondichter hinreichend, ihm anzuzeigen, daß ich von ihm bloß ein Lied erwarte. "18

Es ist offensichtlich, dass Tomášek durch seine Vertonungen von Goethes Gedichten dessen Forderungen erfüllt hat. Das bedeutet aber nicht, dass sein Liedschaffen in allen Aspekten mit dem Stil der Zweiten Berliner Liederschule übereinstimmt. Er reflektierte zwar zweifellos ihre stilistischen und künstlerischen Impulse, ähnlich aber war es aber auch mit den Einflüssen der Schwäbischen und der Wiener Liederschule. Es ist eine sehr interessante Frage, wie weit sich in seinen Liedern die stilistischen Impulse der erwähnten Liederschulen spiegeln, und wie inwieweit seine Kompositionen Ergebnis seiner einigen Ästhetik sind, zu deren Ausgangspunkten die klassisch-reinen Linien der Komposition, Form und Harmonie sowie der Respekt gegenüber dem Charakter der vertonten Poesie gehören. Zugunsten des angemessenen und wahrhaften musikalischen Ausdrucks der Gedichte benutzte er aber auch solche Elemente, die ihm eher fremd

17 KIENZLE, Ulrike. Goethe, Johann Wolfgang von. In Die Musik in Geschichte und Gegenwart, Personenteil, Bd. 7, 2nd edition, Kassel et al, 2002, col. 1192-1213.

18 TOMÁŠEK, Václav Jan. Selbstbiographie IV. Libussa 1850, s. 331. 
waren, wie Tonmalerei oder progressive aperiodische melodische Wendungen. Es ist evident, dass Tomášeks Liederschaffen eine sehr beachtenswerte Erscheinung darstellt, die man auch wegen ihres Kontextes mit den ästhetischen und künstlerischen Strömungen seiner Zeit beachten sollte.

\section{Bibliographie}

BUNZEL, Anja - KUBEČKOVÁ, Barbora. Václav Jan Tomášek (1774-1850): A Versatile Lieder Composer? A Comparative Analysis of Selected Goethe Settings by Carl Friedrich Zelter, Václav Jan Tomášek and Johanna Kinkel. Musicologica Olomucensia 20, 2014, S. 15-37.

DEHNER, Jan. Vliv literárních inspirací na genezi romantického strukturování a myšlení v díle V. J. Tomáška (analytický pokus). Opus musicum 9, 1977, Nr. 7, S. 207-215.

FUKAČ, Jiří. Tomášeks früher Beitrag zur musikalischen Metakritik. Studien zur Musikwissenschaft 49, 2002, S. 115-122.

KABELKOVÁ, Markéta. Václav Jan Tomášek (1774-1850). Život a dílo. Disertační práce, Praha: FF UK, 2012.

KIENZLE, Ulrike. Goethe, Johann Wolfgang von. In Die Musik in Geschichte und Gegenwart, Personenteil, Bd. 7, 2nd edition, Kassel et al, 2002, Sp. 1192-1213.

NĚMEC, Zdeněk. Vlastni životopis Václava Jana Tomáška. Praha: Topičova edice, 1941.

PEČMAN, Rudolf. Tendenz zur Klassik: Betrachtungen über V. J. Tomášek. In Sbornik praci Filozofické fakulty brněnské univerzity, H 28, 1993, S. 55-67.

STEIN, Jack M. Was Goethe Wrong about the nineteenth-century Lied? An Examination of the Relation Poem and Music. In PMLA, Vol. 77, č. 3, 1962, S. 232-239. JSTOR. URL: www.jstor. org/stable/460482.

STRAKOVÁ, Theodora. Tomáškovy písně na Goetheho texty. Časopis Moravského muzea 1955, 40, S. 214-252.

ŠŤASTNÁ, Kateřina Alexandra. Tomášek Václav Jan Křtitel. In Český hudebni slovnik osob a instituci [online]. URL: https://www.ceskyhudebnislovnik.cz/slovnik/index.php?option=com_mdictionary\&task=record.record_detail\&id $=3160$.

TARANTOVÁ, Marie. Václav Jan Tomášek. Praha: Orbis, 1946.

TOMÁŠEK, Václav Jan. Selbstbiographie I-IV. Libussa 4-7, 1845-1850.

VÍT, Petr. Estetické myšleni o hudbě. Praha: Academia, 1987.

VÍT, Petr. Zur Frage der Abwandlungen der musikästhetischen Begriffe in den böhmischen Ländern. Acta Musicologica 60, Bd. 2, 1988, S. 195-204. JSTOR. URL: www.jstor.org/stable/932791. Accessed 11 June 2020.

VYSLOUŽIL, Jiří. Václav Jan Křtitel Tomášeks Stellung in der tschechischen Musikkultur. In Sbornik praci Filozofické fakulty brněnské univerzity, 1975, S. 7-18.

VYSLOUŽILOVÁ, Milena. Václav Jan Tomášeks Lieder zu tschechischen Texten. In Sbornik praci Filozofické fakulty brněnské univerzity, H9, 1974, S. 47-64. 